\title{
The analgesic efficacy of preoperative ultrasound- guided transversus abdominis plane block in fat harvesting from the lower abdomen under local anesthesia: A preliminary case series
}

Seung Min Kim, Hyokyung Yoo, Byung Jun Kim

Department of Plastic and Reconstructive Surgery, Seoul National University Hospital, Seoul National University College of Medicine, Seoul, Korea
Background Small-volume liposuction can be performed under local anesthesia, but intolerable pain usually occurs during the perioperative period. This study evaluated the analgesic effect of ultrasound-guided transverse abdominis plane (TAP) block prior to harvesting a small volume of fat from the lower abdomen under local anesthesia and to demonstrate the safety and efficacy of this technique.

Methods Eight patients who participated in a clinical trial of injecting autologous adipose-derived stromal cells obtained from small-volume liposuction of the lower abdomen from May 2020 to July 2020 were included in this study. Ultrasound-guided TAP block was performed 30 minutes before the main procedure. A total of $15 \mathrm{~mL}$ of a mixture of $2 \%$ lidocaine $(20 \mathrm{~mL})$ and normal saline $(10 \mathrm{~mL})$ was injected into the fascia layer between the internal oblique muscle and transverse abdominis muscle. Subjective pain was evaluated through questionnaires by a verbal numerical rating scale (VNRS) at the time of tumescent infiltration and 1, 2, 4, and 6 hours after the operation.

Results The median time required for TAP block was 14 minutes (range, 10-21 minutes). The median VNRS score was 3.0 at the time of tumescent solution infiltration, 1.5 at postoperative 1 hour and 2 hours, and 2.0 at postoperative 4 and 6 hours. No complications occurred in relation to the TAP block procedure, including infection, inflammation, hematoma, nerve palsy, or bowel perforation.

Conclusions Ultrasound-guided TAP block prior to small-volume liposuction of the lower abdomen was confirmed to be a safe and effective technique for perioperative analgesia.

Keywords Transverse abdominis plane / Liposuction / Local anesthesia

\section{INTRODUCTION}

Liposuction is one of the most commonly performed aesthetic sur-

Received: Aug 4, 2020 Revised: Sep 10, 2020 Accepted: Oct 8, 2020 Correspondence: Byung Jun Kim Department of Plastic and Reconstructive Surgery, Seoul National University Hospital, Seoul National University College of Medicine, 101 Daehak-ro, Jongno-gu, Seoul 03080, Korea Tel: +82-2-2072-2370, Fax: +82-2-2072-0274, E-mail: bjkim79@gmail.com

Copyright @ 2021 The Korean Society for Aesthetic Plastic Surgery.

This is an Open Access article distributed under the terms of the Creative Commons Attribution Non-Commercial License (https://creativecommons.org/licenses/by-nc/4.0/) which permits unrestricted non-commercial use, distribution, and reproduction in any medium, provided the original work is properly cited. $\quad w w w . e-a a p s . o r g$ gical procedures, and it can be classified into four types according to the volume of infiltration: dry, wet, superwet, and the tumescent technique. In the tumescent technique, a large volume of diluted local anesthetic solution is infiltrated into the fat layer to facilitate anesthesia and reduce blood loss. Unlike high-volume liposuction, which usually requires general anesthesia or monitored anesthesia care (MAC), liposuction of a relatively small volume, such as a fat graft for sunken eyelids or minor correction of deformed breasts after major reconstructions, can be performed under local anesthesia [1].

No single anesthetic technique has been proven to be superior over another in liposuction. Avoiding general anesthesia or MAC helps to reduce possible morbidities of the respiratory or circulato- 
ry system, including thromboembolism, pulmonary edema, fat embolism, and cardiac arrest [2-4]. However, it also has the shortcoming of intolerable pain during and after surgery. Based on our previous experiences, infiltrating the tumescent solution into the field of liposuction alone was not sufficient to control perioperative pain in most cases under local anesthesia. The pain was especially intolerable during the process of tumescent cannula dissection.

Therefore, we considered an additional regional block technique to alleviate intraoperative and postoperative pain in patients undergoing liposuction under local anesthesia. Transverse abdominis plane (TAP) block is a regional anesthetic tool to reduce pain in the lower abdomen by administering a local solution into the fascia layer between the internal oblique muscle (IOM) and transversus abdominis muscle (TAM). It has been found to be safe and effective for the management of postoperative pain after abdominoplasty, cesarean section, and laparoscopic-assisted intra-abdominal surgery under general anesthesia [5-9]. However, to the best of our knowledge, no previous studies have evaluated the use of TAP block prior to liposuction under local anesthesia.

This study aimed to evaluate perioperative pain in patients undergoing ultrasound-guided TAP block before abdominal liposuction of a small volume of fat under local anesthesia and to demonstrate the safety and efficacy of this anesthetic technique.

\section{METHODS}

Patients who participated in a clinical trial evaluating the efficacy of intravenous injections of autologous adipose-derived stromal cells (ADSCs) obtained from small-volume liposuction of the lower abdomen from May 2020 to July 2020 were included in this study.

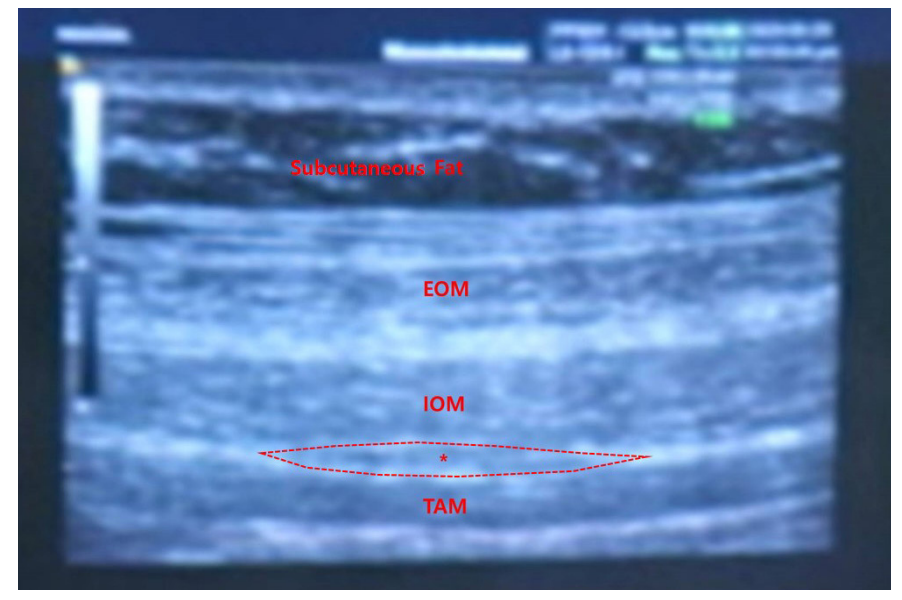

Fig. 1. The different layers of skin, subcutaneous tissue, external oblique muscle (EOM), internal oblique muscle (IOM), and transverse abdominis muscle (TAM) were identified by ultrasonography. The asterisk indicates the target layer of tumescent injection between the IOM and TAM.
This study was approved by the Institutional Review Board of Seoul National University Hospital (IRB Nos. 2003-219-1114, 2001-0971096) and was performed in accordance with the recommendations of the Declaration of Helsinki for biomedical research involving human subjects. Written informed consent was obtained from all participants, who were informed about the purpose, procedures, potential effects, and complications of the procedures performed in the study.

The TAP block was performed under ultrasound guidance by a single surgeon 30 minutes prior to the main procedure in a separate room next to the ambulatory operating room where liposuction was performed. After skin preparation, the different layers of the skin, subcutaneous tissue, and the underlying muscles, including the external oblique muscle (EOM), IOM, and TAM, with distinct fascia layers in between, were identified by applying an aseptic ultrasound probe (Accuvix XQ; Medison, Hwaseong, Korea) at the anterior axillary line (Fig. 1).

By holding the probe at the lateral side of the abdomen and gently exerting pressure in the medial direction with the non-dominant hand, the target layer of the fascia was clearly visualized (Fig. 2). A 70-mm-long 25-gauge spinal needle (Becton, Dickinson and Company, Franklin Lakes, NJ, USA) was smoothly inserted and gently advanced into the muscle layers using the dominant hand. A popping sensation was felt as the needle entered the fascia between the EOM and IOM, and a second popping sensation was felt as the needle entered the fascia between the IOM and TAM. After verifying that the end of the needle was located in the target layer between the IOM and TAM on ultrasonography and confirming that blood did not regurgitate when negative pressure was applied to the spinal needle syringe, $1 \mathrm{~mL}$ of prepared local solution was

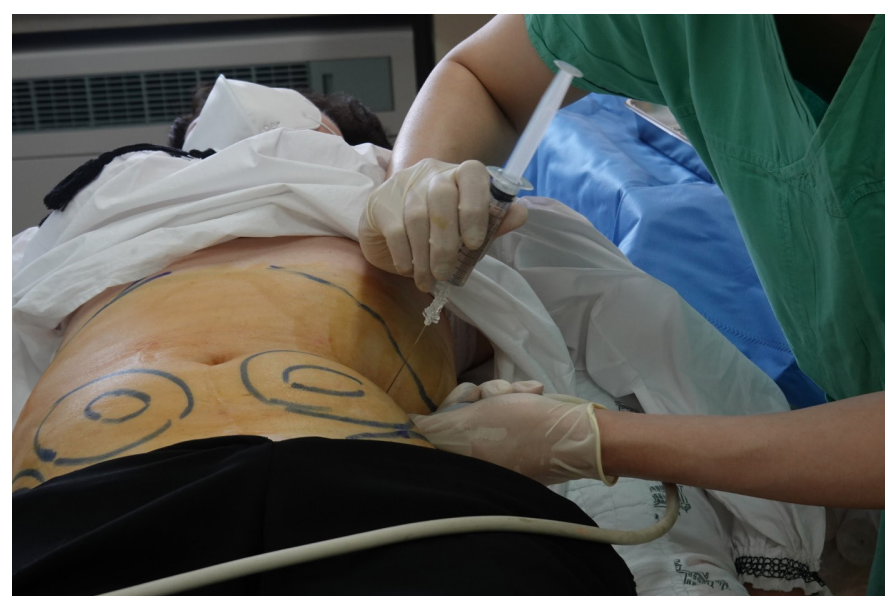

Fig. 2. After identifying the target layer by holding the ultrasound probe at the lateral side of the abdomen and gently exerting pressure in the medial direction with the non-dominant hand, the local solution is injected into the fascia layer between the internal oblique muscle and transverse abdominis muscle with the dominant hand. 
injected to ensure that the target layer was inflated. A total of 15 $\mathrm{mL}$ of a mixture of $2 \%$ lidocaine $(20 \mathrm{~mL}$; Daihan Pharm. Co. Ltd., Seoul, Korea) and normal saline $(10 \mathrm{~mL})$ was slowly injected on each side of the lower abdomen.

After 30 minutes, $0.2 \mathrm{~mL}$ of $2 \%$ lidocaine was infiltrated around the pubic symphysis and the tumescent-infiltrating cannula was inserted through a slit incision. The space for tumescent infiltration was obtained by extensive subcutaneous dissection using the cannula, and a total of $90 \mathrm{~mL}$ of tumescent solution (1 L of normal saline $+25 \mathrm{~mL}$ of $2 \%$ lidocaine $+5 \mathrm{mEq}$ of sodium bicarbonate in 5 $\mathrm{mL}+1 \mathrm{mg}$ of epinephrine in $1 \mathrm{~mL}+3,000 \mathrm{IU}$ of hyaluronidase in 2 $\mathrm{mL}$ ) was injected throughout the lower abdomen. Subjective pain at this point was recorded to evaluate the initial analgesic effect of the TAP block. After 10 minutes, liposuction was performed using 10 $\mathrm{mL}$ luer-lock syringes (Becton, Dickinson and Company) and Mercedes cannulas (MicroAire Surgical Instruments, Charlottesville, VA, USA). When a sufficient amount of fat was harvested, the slit incision wound was repaired using 5-0 Prolene (Johnson \& Johnson Medical Devices, New Brunswick, NJ, USA) and a compressive dressing was placed. The $20-30 \mathrm{~mL}$ of fat harvested from the lower abdomen was sent to the research center to obtain autologous ADSCs.

Subjective pain was evaluated through patient questionnaires using a verbal numerical rating scale (VNRS; 0 , no pain; 10 , the worst possible pain) at the time of tumescent cannula dissection, and 1,2, 4, 6 hours after the operation.

\section{RESULTS}

There were a total of eight patients, including three diagnosed with refractory atopic dermatitis and five with severe osteoarthritis of the knee joints, who underwent liposuction from the lower abdomen (Table 1). Two patients were men and six were women, with a median age of 57 years (range, 20-75 years). All patients were pre- viously healthy with no underlying diseases or previous surgical history except for one patient with a history of laparoscopic appendectomy.

The median time required for the TAP block was 14 minutes (range, 10-21 minutes). The median VNRS score was 3.0 at the time of tumescent cannula dissection and infiltration, 1.5 at postoperative 1 hour and 2 hours, and 2.0 at postoperative 4 and 6 hours. The median VNRS score was 3.0 at the time of tumescent cannula dissection and infiltration, 1.5 at postoperative 1 hour and 2 hours, and 2.0 at postoperative 4 and 6 hours (Fig. 3). In seven of the eight patients, the perioperative VNRS scores were $\leq 3$, indicating mild tolerable pain, even during tumescent cannula dissection. In one patient (case 7), moderate pain (VNRS: 6) persisted during the operation and began to improve 2 hours postoperatively. Subjective discomfort showed a tendency to increase after 4 hours in some cases, although it was not significant. There were no cases involving complications related to the TAP block procedure, including infection, inflammation, hematoma, nerve palsy, or bowel perforation.

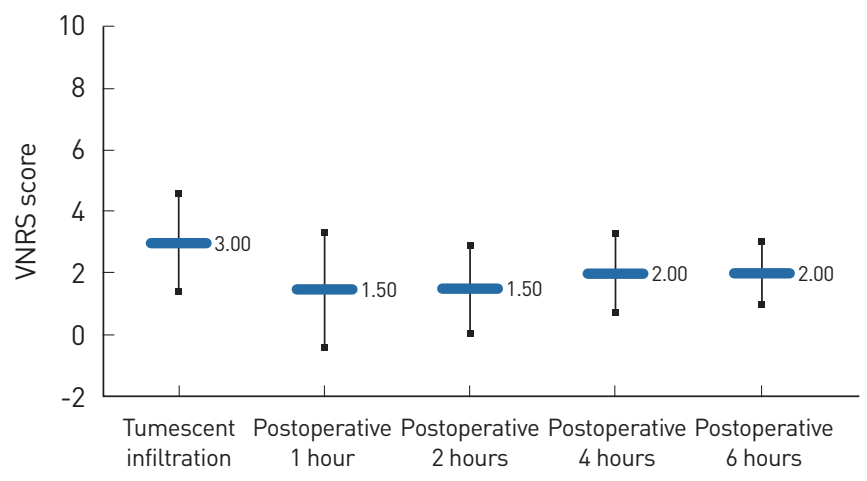

Fig. 3. Intraoperative and postoperative verbal numerical rating scale (VNRS) scores of eight patients.

Table 1. Patient demographics and VNRS scores

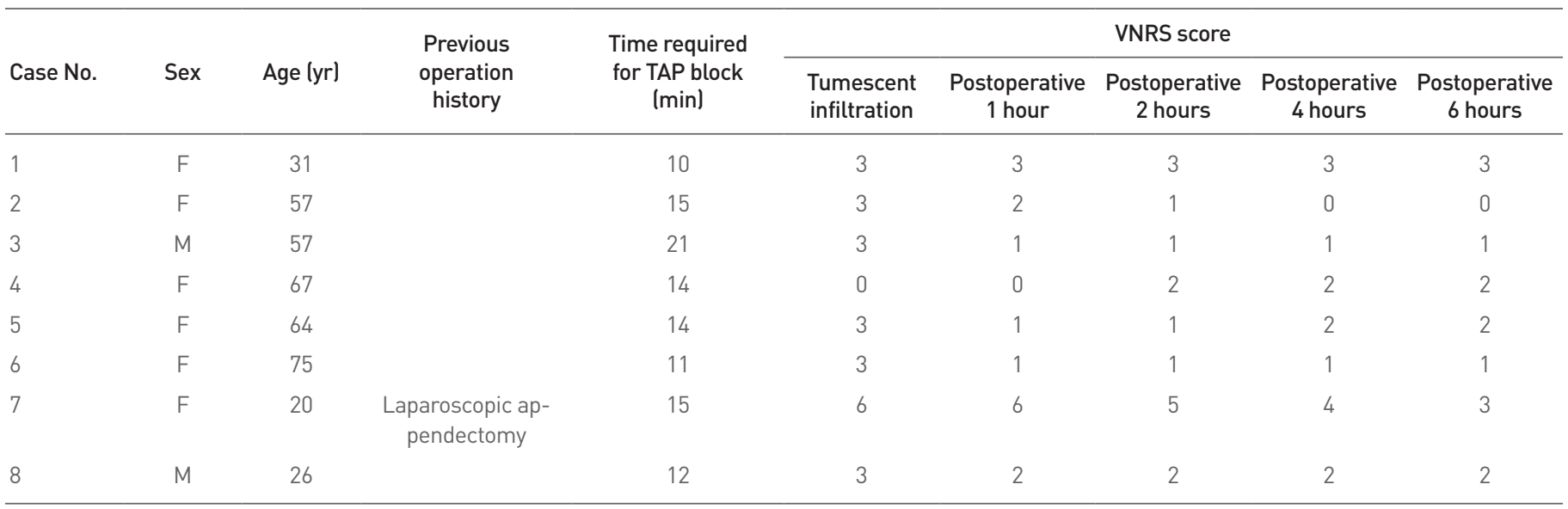

VNRS, verbal numerical rating scale; TAP, transverse abdominis plane; F, female; $M$, male. 


\section{DISCUSSION}

The ultrasound-guided TAP block is a regional block technique for analgesia of the lower abdomen through the local injection of solutions to block T7 to T12 and L1, as well as the iliohypogastric nerve, ilioinguinal nerve, subcostal nerve, and intercostal nerve [10]. The technique was first introduced in 1993 by Kuppuvelumani et al. [11] who successfully blocked T10-L1 with local anesthetics to relieve postoperative pain in 30 patients undergoing cesarean sections. This technique is known to be effective for controlling postoperative pain in a variety of general, gynecological, urological, and plastic abdominal surgical procedures under general anesthesia [5-9].

However, the previous literature on the TAP block prior to liposuction under local anesthesia is very limited. In this study, seven of eight patients who received a TAP block preoperatively before small-volume liposuction under local anesthesia showed VNRS scores of $\leq 3$ from the time of tumescent infiltration to 6 hours after the operation. The anesthetic procedure was safely and quickly performed under direct visual guidance via ultrasonography to ensure accurate local deposition of the solution in the correct neurovascular plane. This technique resulted in a tolerable amount of pain both intraoperatively and postoperatively, without any related complications, such as hematoma, injury of internal organs, and transient palsy of unintended nerves. This technique particularly alleviated discomfort during tumescent cannula dissection, which is when most patients complain of pain during local surgery. The need for administering additional analgesics during the operation was decreased, reducing the adverse effects related to opioids [12]. Overall morbidity was also reduced by avoiding general anesthesia and MAC.

In this study, we locally administered $15 \mathrm{~mL}$ of solution on each side of the abdomen with a mixture of $20 \mathrm{~mL}$ of $2 \%$ lidocaine and $10 \mathrm{~mL}$ of normal saline. This is consistent with some previous studies suggesting that $15 \mathrm{~mL}$ may be an optimal volume for TAP block $[13,14]$. Lidocaine was used as our local anesthetic regimen for its early onset and intermediate duration of analgesic effect, as well as its minimal central nervous system toxicity compared to other regimens, such as ropivacaine, bupivacaine, or levobupivacaine [1517]. For liposuction of a small amount, which usually requires less than 2 hours of surgery, the duration of the effect of lidocaine, which has a half-life of 1.5-2 hours, is sufficient to provide appropriate pain control [18]. In a previous study by Song et al. [19], the effect of TAP block after preoperatively administering $30 \mathrm{~mL}$ of $2 \%$ lidocaine in lower abdominal surgery was statistically significant up to 1 hour postoperatively and showed better analgesic control up to 6 hours after the operation, even though the latter effect was not statistically significant.

For surgical procedures with a longer duration, adding epinephrine into the local solution can prolong the local anesthetic effect and reduce systemic absorption by decreasing local blood flow and slowing lidocaine elimination from the injection site [20,21]. Ropivacaine is also known to provide a significantly prolonged duration of pain control $[22,23]$. Further studies with larger samples can help identify the optimal regimen and dose for surgical procedures of varying duration and extent.

In the exceptional case of one patient with a VNRS score higher than 3, the patient complained of intolerable pain from the tumescent cannula dissection until 2 hours after the operation. This was mostly because the surgical procedure was delayed for 2 hours after the TAP block because the operating room was unavailable.

This study has several limitations, including an insufficient sample size and the lack of a control group, which would be needed to provide a high level of evidence on the effect of TAP block in smallvolume liposuction. However, numerous previous studies have shown that some patients are not able to tolerate intraoperative pain during liposuction under local anesthesia and have suggested the use of the tumescent technique combined with monitored intravenous sedation [24-26]. Further prospective studies with larger samples and control groups are needed to confirm the efficacy of this procedure, as well as the extent and duration of the analgesic effect. Studies should also be conducted to determine the most suitable regimen, dose, concentration, and timing of the injection.

In addition, this study only aimed to evaluate the efficacy of the TAP block in small-volume liposuction under local anesthesia. Pain and discomfort associated with surgical procedures usually depend on the amount of fat harvested because of the need to suction a broad extent of fat, including the deep subcutaneous fat tissue, and the prolonged operation time associated with large-volume liposuction. For large-volume liposuction, general anesthesia is generally recommended due to the possibility of major hemodynamic changes. On the contrary, many cases of small-volume liposuction are performed under local anesthesia with the tumescent technique or under light intravenous sedation $[1,27,28]$. The potential use of the same procedure in intermediate- to large-volume liposuction could be assessed through further studies in order to evaluate the efficacy of TAP block for managing more severe degrees of pain.

In conclusion, ultrasound-guided TAP block with $2 \%$ lidocaine solution 30 minutes prior to small-volume liposuction of the lower abdomen is a safe and effective technique for both intraoperative and postoperative analgesia.

\section{NOTES}

\section{Conflict of interest}

No potential conflict of interest relevant to this article was reported.

\section{Ethical approval}

The study was approved by the Institutional Review Board of Seoul National University Hospital (IRB Nos. 2003-219-1114, 2001-097- 
1096) and performed in accordance with the principles of the Declaration of Helsinki.

\section{Patient consent}

The patients provided written informed consent for the publication and the use of their images.

\section{ORCID}

\section{Seung Min Kim}

Hyokyung Yoo

Byung Jun Kim https://orcid.org/0000-0003-2310-2125

https://orcid.org/0000-0002-9171-0379

https://orcid.org/0000-0002-6891-3768

\section{REFERENCES}

1. Sood J, Jayaraman L, Sethi N. Liposuction: anaesthesia challenges. Indian J Anaesth 2011;55:220-7.

2. Rao RB, Ely SF, Hoffman RS. Deaths related to liposuction. N Engl J Med 1999;340:1471-5.

3. Teimourian B, Rogers WB 3rd. A national survey of complications associated with suction lipectomy: a comparative study. Plast Reconstr Surg 1989;84:628-31.

4. Gilliland MD, Coates N. Tumescent liposuction complicated by pulmonary edema. Plast Reconstr Surg 1997;99:215-9.

5. Araco A, Pooney J, Araco F, et al. Transversus abdominis plane block reduces the analgesic requirements after abdominoplasty with flank liposuction. Ann Plast Surg 2010;65:385-8.

6. Sforza M, Andjelkov K, Zaccheddu R, et al. Transversus abdominis plane block anesthesia in abdominoplasties. Plast Reconstr Surg 2011; 128:529-35.

7. Belavy D, Cowlishaw PJ, Howes M, et al. Ultrasound-guided transversus abdominis plane block for analgesia after Caesarean delivery. Br J Anaesth 2009;103:726-30.

8. Albrecht E, Kirkham KR, Endersby RV, et al. Ultrasound-guided transversus abdominis plane (TAP) block for laparoscopic gastric-bypass surgery: a prospective randomized controlled double-blinded trial. Obes Surg 2013;23:1309-14.

9. Sivapurapu V, Vasudevan A, Gupta S, et al. Comparison of analgesic efficacy of transversus abdominis plane block with direct infiltration of local anesthetic into surgical incision in lower abdominal gynecological surgeries. J Anaesthesiol Clin Pharmacol 2013;29:71-5.

10. Mishra M, Mishra SP. Transversus abdominis plane block: the new horizon for postoperative analgesia following abdominal surgery. Egypt J Anaesth 2016;32:243-7.

11. Kuppuvelumani P, Jaradi H, Delilkan A. Abdominal nerve blockade for postoperative analgesia after caesarean section. Asia Oceania J Obstet Gynaecol 1993;19:165-9.

12. White PF. The changing role of non-opioid analgesic techniques in the management of postoperative pain. Anesth Analg 2005;101:S5-22.
13. Abdallah FW, Chan VW, Brull R. Transversus abdominis plane block: a systematic review. Reg Anesth Pain Med 2012;37:193-209.

14. Moeschler SM, Murthy NS, Hoelzer BC, et al. Ultrasound-guided transversus abdominis plane injection with computed tomography correlation: a cadaveric study. J Pain Res 2013;6:493-6.

15. Hyndman J. Pharmacology for regional anaesthesia [Internet]. London: Anaesthesia Tutorial of the Week; c2007 [cited 2020 Nov 18]. Available from: http://e-safe-anaesthesia.org/e_library/09/Pharmacology_ for_regional_anaesthesia_TOTW_049_2007.pdf.

16. Guinet P, Estebe JP, Ratajczak-Enselme M, et al. Electrocardiographic and hemodynamic effects of intravenous infusion of bupivacaine, ropivacaine, levobupivacaine, and lidocaine in anesthetized ewes. Reg Anesth Pain Med 2009;34:17-23.

17. Reiz S, Haggmark S, Johansson G, et al. Cardiotoxicity of ropivacaine: a new amide local anaesthetic agent. Acta Anaesthesiol Scand 1989;33: 93-8.

18. Estebe JP. Intravenous lidocaine. Best Pract Res Clin Anaesthesiol 2017; 31:513-21.

19. Song IK, Kim CH, Han JI, et al. The analgesic efficacy of ultrasoundguided transversus abdominis plane block with $2 \%$ lidocaine in early postoperative period after lower abdominal surgery. Anesth Pain Med 2012;7:280-5.

20. Bernards CM, Kopacz DJ. Effect of epinephrine on lidocaine clearance in vivo: a microdialysis study in humans. Anesthesiology 1999;91:962-8.

21. Rahiri J, Tuhoe J, Svirskis D, et al. Systematic review of the systemic concentrations of local anaesthetic after transversus abdominis plane block and rectus sheath block. Br J Anaesth 2017;118:517-26.

22. Vaddi P, Chakravarthy K, Kotla HB, et al. A comparative study of $0.5 \%$ ropivacaine vs $0.25 \%$ bupivacaine in transverse abdominis plane block for post operative analgesia in patients undergoing abdominal surgeries J Med Sci Clin Res 2019;7:723-30.

23. Fuladi N, Deshmukh S, Bhure A. Comparative study of bupivacaine $0.25 \%$ versus ropivacaine $0.5 \%$ in transversus abdominis plane block for postoperative analgesia in lower abdominal surgeries: a randomised controlled trial. J Evol Med Dent Sci 2014;3:4569-77.

24. Pitman GH, Aker JS, Tripp ZD. Tumescent liposuction: a surgeon's perspective. Clin Plast Surg 1996;23:633-41.

25. Scarborough DA, Herron JB, Khan A, et al. Experience with more than 5,000 cases in which monitored anesthesia care was used for liposuction surgery. Aesthetic Plast Surg 2003;27:474-80.

26. Narins RS, Coleman WP 3rd. Minimizing pain for liposuction anesthesia. Dermatol Surg 1997;23:1137-40.

27. Kim YK, Lee SJ. Correction of sunken upper eyelids using autologous microfat graft. J Korean Soc Aesthetic Plast Surg 2006;12:79-82.

28. Glasgold RA, Lam SM, Glasgold MJ. Periorbital fat grafting. In: Massry GG, Murphy MR, Azizzadeh B, editors. Master techniques in blepharoplasty and periorbital rejuvenation. New York: Springer; 2011. p. 25972. 